\title{
The Norwegian Sequencing Centre [NSC]
}

\section{Robert Lyle, Tim Hughes, NSC, Dag Undlien, Kjetill Jakobsen}

Department of Medical Genetics and Norwegian Sequencing Centre Oslo University Hospital, Kirkeveien 166, 0407, Oslo, Norway

http://www.sequencing.uio.no/

The Norwegian Sequencing Centre (NSC) was established in 2009 to provide the Norwegian research community with access to high-throughput sequencing services. Currently, 16 people work at the NSC. Funding has been provided from a number of sources, including Health South-East, the University of Oslo, and the Norwegian Research Council.

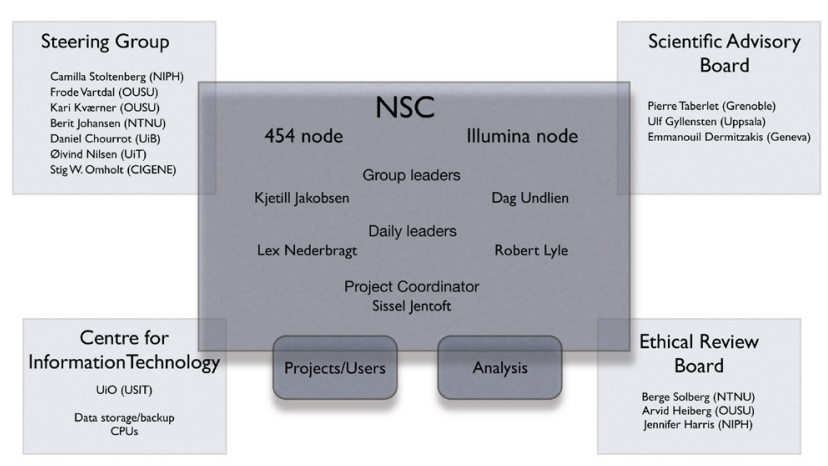

Two main activities of the NSC will contribute to the aims of the SEQAHEAD project. 1. Experience providing a very broad range of sequencing applications based on a range of technology platforms. 2. The development of a national storage and analysis platform for human genetic data.

1. The NSC has 454 (GS FLX+), Illumina (GAllx, HiSeq) and Pacific Biosciences (RS) platforms. In addition Illumina MiSeq and Ion Torrent (PGM) machines have been ordered. This enables us to support a broad range of projects and sequencing applications. This includes large scale de novo projects, such as the cod genome (doi:10.1038/naturel0342), transcriptomics (mRNA, miRNA), epigenetics (RRBS, ChIP), and resequencing (exomeseq).

2. The medical genetics department at the Oslo University Hospital has initiated and received funding for the development of a national storage and analysis platform for DNA sequence data to be used by the Norwegian health service. Partners in the project are the University High Performance Computing unit, the Informatics Department, and the hospital IT and Data Protection units. The system should enable the secure transfer of sequence data and meta-data from production sites to the system, strict access control functionality, secure communication between users of the system, and interfaces for power users (e.g. bioinformaticians and medical genetics clinicians) and expert computer systems (e.g. pharmaco-genetics expert system). In addition, the system needs to be highly scalable to accomodate what is anticipated to be the explosive use of genetic information in the treatment of a broad range of pathologies. The above requirements will require the design and development of a secure high performance computing infrastructure that not only satisfies the technical requirements, but also complies with the strict data security laws that apply to sequence data in Norway. In addition, secure data software services will need to be developed and run on top of this infrastructure. The goal is to have a working pilot of the system installed by the spring of 2015.

\section{Relevant Web sites}

1. http://www.sequencing.uio.no/

2. http://codgenome.no/

3. https://wiki.uio.no/usit/suf/vd/hpc/index.php/Tsd 